\title{
Increased incidence of Mycoplasma pneumoniae infections detected by laboratory-based surveillance in Denmark in 2010
}

J N Rasmussen ${ }^{1}$, M Voldstedlund ${ }^{1}$, R L Andersen², S Ellermann-Eriksen ${ }^{3}$, T G Jensen ${ }^{4}$, H K Johansen ${ }^{5}$, B Kolmos ${ }^{6}$, M Mølvadgaard $^{7}$, S S Nielsen ${ }^{1}$, E Olsen ${ }^{8}, K_{\text {S Schønning }}{ }^{,}$S A Uldum (su@ssi.dk)

1. Statens Serum Institut, Copenhagen, Denmark

2. Clinical Microbiology Department, Nordsjællands Hospital, Hillerød, Denmark

3. Clinical Microbiology Department, Århus University Hospital, Skejby, Denmark

4. Clinical Microbiology Department, Odense University Hospital, Odense, Denmark

5. Clinical Microbiology Department, Rigshospitalet, Copenhagen, Denmark

6. Clinical Microbiology Department, Sygehus Lillebælt, Vejle, Denmark

7. Clinical Microbiology Department, Aalborg Sygehus Syd, Aalborg, Denmark

8. Clinical Microbiology Department, Regionshospitalet Viborg, Skive,Viborg, Denmark

9. Clinical Microbiology Department, Hvidovre Hospital, Hvidovre, Denmark

Citation style for this article:

Rasmussen JN, Voldstedlund M, Andersen RL, Ellermann-Eriksen S, Jensen TG, Johansen HK, Kolmos B, Mølvadgaard M, Nielsen SS, Olsen E, Schønning K, Uldum SA. Increased incidence of Mycoplasma pneumoniae infections detected by laboratory-based surveillance in Denmark in 2010 . Euro Surveill.

2010;15(45): $\mathrm{pii}=19708$. Available online: $h$ ttp://www.eurosurveillance.org/ViewArticle. aspx?Articleld =19708

In Denmark recurrent epidemics of Mycoplasma pneumoniae infections have been described since the 1950 s at intervals of approximately four to six years. The latest epidemic occurred in 2004/05 followed by two years of high incidence and more than three years of low incidence. Due to a recent increase in diagnosed cases since late summer 2010, we conducted a survey of positive $M$. pneumoniae PCR tests performed by clinical microbiology departments in Denmark, which indicated that a new epidemic may be underway.

\section{Introduction}

Mycoplasma pneumoniae is a common cause of upper and especially lower respiratory tract infections such as bronchitis and pneumonia. In addition, M. pneumoniae causes neurological symptoms and sequelae in a high proportion of cases $[1,2]$. The highest prevalence is seen in children and younger adults. Cases occur throughout the year, but the incidence is highest during autumn and winter. In Denmark, regular epidemics have been described since 1949/50. With the exception of a nine-year endemic period from 1978 to 1987 [3], these epidemics usually begin during summer, culminate in late autumn/early winter and fade out during winter. In some instances the epidemics span two winters: this was seen in 1962 to 1964 and 1971 to 1973 [3]. The latest epidemic in 2004/05 [4,5] was followed by two years of high incidence, but since 2007 the incidence has been very low judging by the low rate of on average approximately $3 \%$ positive samples seen in this period (Figure 1 ).

From 1946 until the late 1990s the central national laboratory at Statens Serum Institut (SSI) received samples from the whole country for the diagnosis of $M$. pneumoniae infections [3]. In the last decades the local clinical microbiology departments have taken over a large part of the laboratory tests for M. pneumoniae. The diagnosis had previously been based on serology but since the beginning of the $1990 \mathrm{~s}$ PCR has been introduced as a routine diagnostic test at SSI for rapid and early diagnosis of $M$. pneumoniae infection [6], and in more recent years, most of the local departments have also adopted PCR. The countrywide use of PCR for diagnosis and surveillance of M. pneumoniae infections is probably unique for Denmark.

Although $\mathrm{SSI}$ is now predominantly receiving samples from the eastern part of the country only, the institute is the one laboratory in Denmark performing most tests for M. pneumoniae overall, and thus results obtained at SSI may be seen as indicative of the M. pneumoniae activity in Denmark as a whole. Each week the rate of positive samples is calculated, and a rise from approximately $5 \%$ to $15 \%$ or more positive samples within approximately six weeks are considered as indicative of an M. pneumoniae epidemic [4].

At SSI we saw an increase in the number of positive samples above the threshold in the beginning of October 2010. This prompted us to investigate whether this was the beginning of an epidemic of M. pneumoniae infections in Denmark in the autumn of 2010.

\section{Methods}

Because PCR is found superior to serology for the diagnosis of $M$. pneumoniae infection during the early phases of infection [7], we included in our investigation only those records that were diagnosed by a PCR-based method. The departments use a range of different PCR 
assays, of which some are published $[6,8,9]$ or commercial kits, but most are unpublished but validated in-house assays.

A survey was conducted collecting data from all clinical microbiology departments in Denmark performing PCR testing for M. pneumoniae for general practitioners and hospitals. In addition to SSI, there are 12 such departments in the country that perform this analysis and we received data from 11 of them. They represented all five regions in Denmark (Figure 2): Capital Region of Denmark (data from three of four departments), Region Zealand (data from the sole department), Region of Southern Denmark (data from three of three departments), Central Denmark Region (data from two of two departments) and North Denmark Region (data from the sole department).

From the local departments we obtained data on the total number of PCR analyses performed and the number of analyses positive for $M$. pneumoniae for week 1 in 2009 to week 41 in 2010. Only data for weeks 34 to 41 in 2009 and 2010 are compared in the analysis presented here. From SSI we obtained data from week 1 in 2004 to week 41 in 2010 (October 16). We present the number of positive tests and the weekly proportion of positive tests among all tests performed. Since the catchment areas of the departments are not well defined, i.e. the general practitioner can send the specimen to any department, it was not possible to calculate the regional incidences. However, the total population of Denmark is 5.5 million and we used this to calculate an estimated incidence of PCR-diagnosed M. pneumoniae.

\section{Results}

Figure 1 shows the M. pneumoniae tests performed at SSI from week 1 in 2004 to week 41 in 2010. From 2007 to 2010 the average positivity rate of $M$. pneumoniae infection in Denmark remained very low, at approximately $3 \%$ positive samples (Figure 1). Apart from a short peak in the number of positive tests observed in week 50 in 2008, the first increase in the positivity rate since 2007 was observed in late August 2010 (weeks 33-35) when it rose to approximately $10 \%$. The rate increased further in the following weeks and reached approximately $15 \%$ in late September/early October (weeks 39-40) despite a three- to fourfold increase in the number of samples received for PCR in this period (Table). This increase in the rate of positive M. pneumoniae tests occurred in all regions, but was seen a little later in the regions than at SSI (Table). The estimated national incidence of PCR-diagnosed M. pneumoniae infections in 2010 rose from 0.4 per 100.000 in week 34 to 3 per 100.000 in week 41.

\section{Discussion and conclusion}

Recurrent epidemics of $M$. pneumoniae infection are also well known in other countries $[10,11]$ and a few reports indicate simultaneous epidemics in more than one country $[12,13]$. M. pneumoniae epidemics have a high impact on the community, and a laboratory-based system for the surveillance of this disease is recommendable. According to our knowledge Denmark is the only country with a PCR-based surveillance system for M. pneumoniae. A rapid increase in macrolide-resistant $M$. pneumoniae has been reported from Asia in the recent years, but macrolide resistance it is also seen in Europe and in the United States [14]. In Denmark SSI did a survey after the epidemic in 2004 and found 1-2\% of macrolide resistance. This is in accordance with a recent German study [15] indicating a limited but not negligible level of resistance in Europe. If an epidemic is recognised it is possible to guide the hospitals and general practitioners in the diagnosis and antibiotic treatment of the disease. Only a focused use of

\section{FIGURE 1}

Mycoplasma pneumoniae PCR tests done at Statens Serum Institut, Denmark, week 12004 - week 41, 2010*

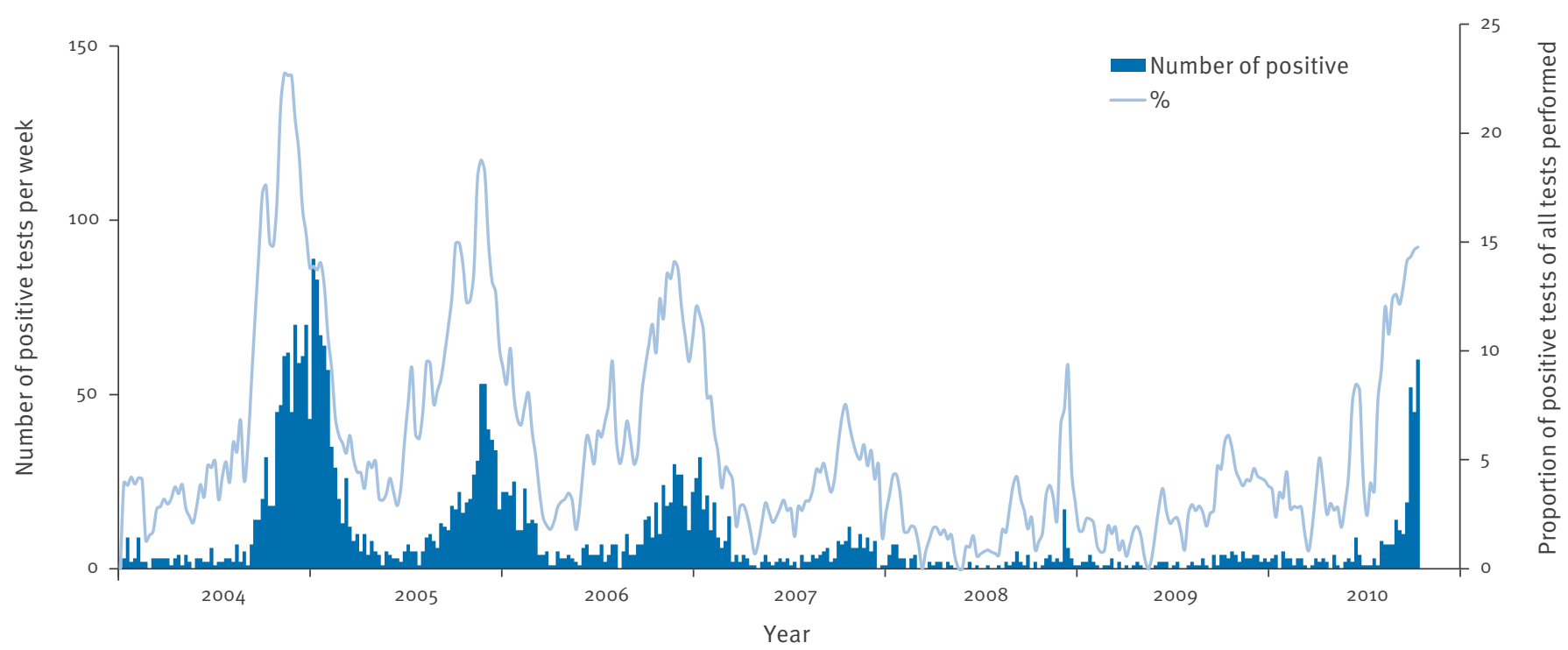

The percentages are the floating average of three weeks. 


\section{FIGURE 2}

The five administrative regions of Denmark and population numbers

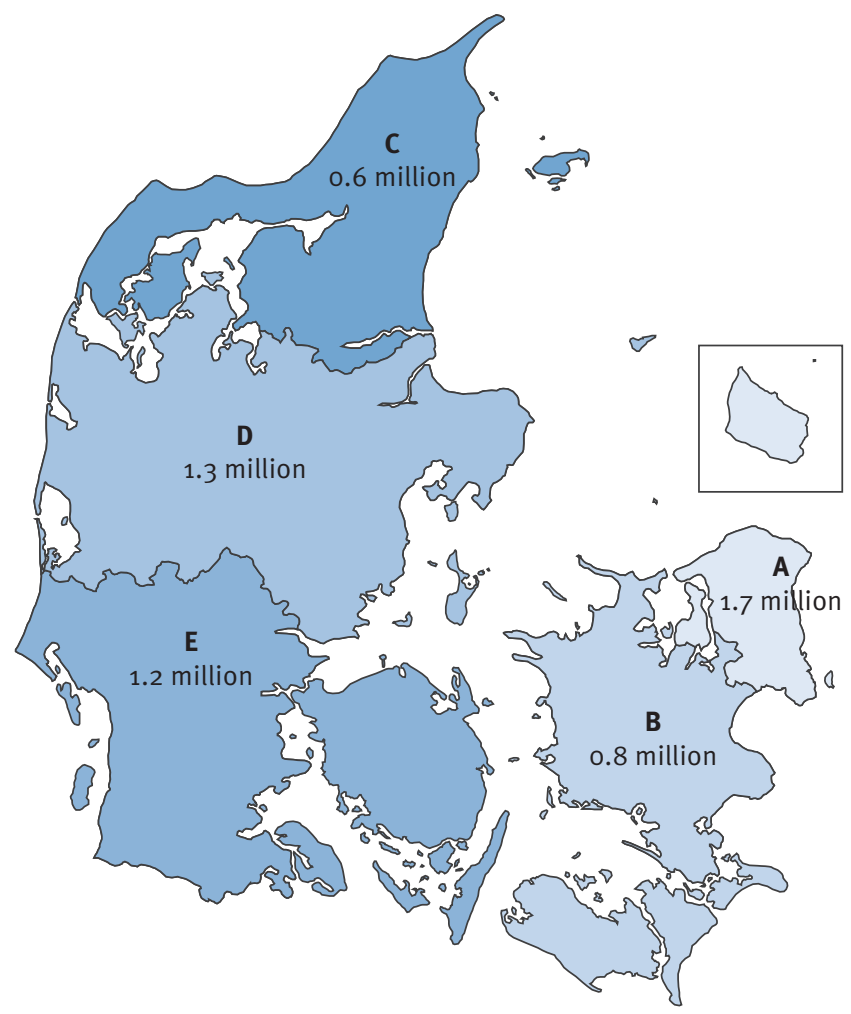

macrolide antibiotics in diagnosed cases can diminish the risk of spreading resistant bacteria.

In conclusion, we have seen an increase in the number of positive tests and also in the positivity rate of submitted samples since late summer 2010, indicating increased transmission of $M$. pneumoniae. The findings suggest that Denmark may be in the early phase of an epidemic. Other European countries, if data are available, should assess if they are in a similar situation.

\section{Acknowledgements}

We acknowledge Dr. Kjeld Truberg Jensen for providing data from Clinical Microbiology Department, Sydvestjysk Sygehus Esbjerg, Denmark.

*Authors' correction:

On request of the authors, Figure 1 was exchanged on 18 November 2010.

A: Capital Region of Denmark; B: Region Zealand; C: North

Denmark Region; D: Central Denmark Region; E: Region of

Southern Denmark

Population 1 July 2010. Source Statistics Denmark

(http://www.dst.dk/HomeUK.aspx).

\section{TABLE}

Number and proportion of positive tests for Mycoplasma pneumoniae performed by Statens Serum Institut and the clinical microbiology departments in the regions, Denmark, 2009 and 2010

\begin{tabular}{|c|c|c|c|c|c|c|c|c|}
\hline \multirow{2}{*}{$\begin{array}{c}\text { Region } \\
\text { and year }\end{array}$} & \multicolumn{8}{|c|}{ Number of positive test and number of all tests performed (\%) } \\
\hline & Week 34 & Week 35 & Week 36 & Week 37 & Week 38 & Week 39 & Week 40 & Week 41 \\
\hline \multicolumn{9}{|l|}{$\mathrm{SSI}^{\mathrm{a}}$} \\
\hline 2009 & 1 of $55(1.8)$ & 3 of $69(4.3)$ & 1 of $70(1.4)$ & o of $66(0)$ & 4 of $61(6.6)$ & 1 of $60(1.7)$ & 4 of $71(5.6)$ & 4 of $66(6.1)$ \\
\hline 2010 & 7 of $68(10.3)$ & 14 of $91(15.4)$ & 11 of $96(11.5)$ & 10 of 101 (9.9) & 20 of $112(17.9)$ & 52 of $362(14.4)$ & 45 of $338(13.3)$ & 60 of $374(16.0)$ \\
\hline \multicolumn{9}{|l|}{ Capital } \\
\hline 2009 & 0 of $30(0)$ & 1 of 34 (2.9) & 5 of $29(17.2)$ & o of $37(0)$ & 1 of $37(2.7)$ & 1 of $49(2.0)$ & 1 of $47(2.1)$ & o of 39 (o) \\
\hline 2010 & 6 of $53(11.3)$ & 5 of $74(6.8)$ & 4 of $84(4.8)$ & 6 of $59(10.2)$ & 3 of $75(4.0)$ & 16 of $233(6.9)$ & 25 of $218(11.5)$ & 24 of $224(10.7)$ \\
\hline \multicolumn{9}{|l|}{ Zealand } \\
\hline 2009 & o of $5(0)$ & o of $12(0)$ & o of $14(0)$ & o of 15 (o) & 1 of $17(5.9)$ & o of $13(0)$ & o of $11(0)$ & o of $11(0)$ \\
\hline 2010 & 2 of $10(20.0)$ & 1 of 11 (9.1) & 3 of 13 (23.1) & 2 of $30(6.7)$ & 3 of $20(15.0)$ & 15 of $86(17.4)$ & 7 of $61(11.5)$ & 20 of $85(23.5)$ \\
\hline \multicolumn{9}{|c|}{ Southern Denmark } \\
\hline 2009 & 2 of $45(4.4)$ & 1 of $37(2.7)$ & 1 of $51(2.0)$ & o of 68 (o) & 1 of $60(1.7)$ & 1 of $57(1.8)$ & 1 of $62(1.6)$ & 1 of $61(1.6)$ \\
\hline 2010 & 2 of $41(4.9)$ & 1 of $40(2.5)$ & 3 of $43(7.0)$ & 2 of $82(2.4)$ & 2 of $81(2.5)$ & 10 of $137(7.3)$ & 20 of $165(12.1)$ & 22 of 189 (11.6) \\
\hline \multicolumn{9}{|c|}{ Central Denmark } \\
\hline 2009 & o of $8(0)$ & o of $16(0)$ & 1 of $17(5.9)$ & 0 of $25(0)$ & 0 of $16(0)$ & o of $13(0)$ & 1 of $16(6.3)$ & 1 of $26(3.8)$ \\
\hline 2010 & 4 of $25(16.0)$ & 1 of $18(5.6)$ & 2 of $23(8.7)$ & 4 of $23(17.4)$ & 1 of $22(4.5)$ & 7 of $53(13.2)$ & 11 of $60(18.3)$ & 10 of $65(15.4)$ \\
\hline \multicolumn{9}{|c|}{ North Denmark } \\
\hline 2009 & o of 6 (o) & 0 of $3(0)$ & o of 8 (o) & o of $10(0)$ & o of $15(0)$ & o of $11(0)$ & 1 of $12(8.3)$ & 1 of $8(12.5)$ \\
\hline 2010 & o of $17(0)$ & 3 of $17(17.6)$ & 1 of $15(6.7)$ & 1 of $14(7.1)$ & 2 of $19(10.5)$ & 4 of $44(9.1)$ & 7 of $55(12.7)$ & 28 of $165(17.0)$ \\
\hline \multicolumn{9}{|l|}{ Total } \\
\hline 2009 & 3 of $149(2.0)$ & 5 of $171(2.9)$ & 8 of $189(4.2)$ & o of 221 (o) & 7 of 206 (3.4) & 3 of $203(1.5)$ & 8 of $219(3.7)$ & 7 of $211(3.3)$ \\
\hline 2010 & 21 of 214 (9.8) & 25 of $251(10.0)$ & 24 of $274(8.8)$ & 25 of 309 (8.1) & 31 of $329(9.4)$ & 104 of $915(11.4)$ & 115 of 897 (12.8) & 164 of 1102 (14.9) \\
\hline
\end{tabular}

a Statens Serums Institut (SSI) receives samples not only from the capital region but also from the rest of the country and is therefore presented separately. 


\section{References}

1. Waites KB, Talkington DF. Mycoplasma pneumoniae and its role as a human pathogen. Clin Microbiol Rev. 2004;17(4):697-728.

2. Tsiodras S, Kelesidis I, Kelesidis T, Stamboulis E, Giamarellou $\mathrm{H}$. Central nervous system manifestations of Mycoplasma pneumoniae infections. J Infect. 2005;51(5):343-54

3. Lind K, Benzon MW, Jensen JS, Clyde WA Jr. A

seroepidemiological study of Mycoplasma pneumoniae infections in Denmark over the 50-year period 1946-1995. Eur J Epidemiol. 1997;13(5):581-6

4. Uldum SA, Jensen JS, Nielsen SS. Increase in M. pneumoniae. EPI-NEWS 2004 (42/43). Available from: http://www.ssi. $\mathrm{dk} /$ English/NewS/EPI-NEWS/ /media/Indhold/EN\%20-\%20 engelsk/EPI-NEWS/2004/pdf/EPI-NEWS\%20-\%202004\%20 $-\% 20 N_{0} \% 2043$.ashx

5. Uldum SA. Epidemic of Mycoplasma pneumoniae. EPI-NEWS 2004 (51). Available from: http://www.ssi.dk/English/ NewS/EPI-NEWS/ /media/Indhold/EN\%20-\%20engelsk/EPINEWS/2004/pdf/EPI-NEWS\%20-\%202004\%20-\%20No\%2051. ashx

6. Jensen JS, Søndergård-Andersen J, Uldum SA, Lind K. Detection of Mycoplasma pneumoniae in simulated clinical samples by polymerase chain reaction. APMIS. 1989;11(11):1046-8

7. Nilsson AC, Björkman P, Persson K. Polymerase chain reaction is superior to serology for the diagnosis of acute Mycoplasma pneumoniae infection and reveals a high rate of persistent infection. BMC Microbiol. 2008,8:93.

8. Pitcher D, Chalker VJ, Sheppard C, George RC, Harrison TG. Real-time detection of Mycoplasma pneumoniae in respiratory samples with an internal processing control. J Med Microbiol. 2006;55(Pt 2):149-55

9. Welti M, Jaton K, Altwegg M, Sahli R, Wenger A, Bille J.

Development of a multiplex real-time quantitative PCR assay to detect Chlamydia pneumoniae, Legionella pneumoniae and Mycoplasma pneumoniae in respiratory tract secretions. Diagn Microbiol Infect Dis. 2003;45(2):85-95.

10. Rastawicki W, Kaluzewski S, Jagielski M, Gierczyski R. Epidemiology of Mycoplasma pneumoniae infections in Poland: 28 years of surveillance in Warsaw 1970-1997. Euro Surveill. 1998;3(10):pii=95. Available from: http://www. eurosurveillance.org/ViewArticle.aspx?Articleld $=95$

11. Ghosh K, Clements GB. Surveillance of Mycoplasma pneumoniae in Scotland 1986-1991. J Infect .1992;13(2):221-7.

12. Pönkä. Occurrence of serological verified Mycoplasma pneumoniae infections in Finland and in Scandinavia in 1970-1977. Scand J Infect Dis. 1980;12(1):27-31.

13. Akehurst C. Mycoplasma epidemics. Euro Surveill. 1998;2(50): pii=1112. Available from: http://www. eurosurveillance.org/ViewArticle.aspx?Articleld=1112

14. Li X, Atkinson TP, Hagood J, Makris C, Duffy LB, Waites KB. Emerging macrolide resistance in Mycoplasma pneumoniae in Children: detection and characterization of resistant isolates. Pediatr Infect Dis J. 2009;28(8):693-6.

15. Dumke R, von Baum H, Lück PC, Jacobs E. Occurrence of macrolide-resistant Mycoplasma pneumoniae strains in Germany. Clin Microbiol Infect. 2010;16(6):613-6. 